\title{
ECFS-DEA: an ensemble classifier-based feature selection for differential expression analysis on expression profiles
}

\author{
Xudong Zhao ${ }^{1 \dagger}$, Qing Jiao ${ }^{1 \dagger}$, Hangyu $\mathrm{Li}^{1+}$, Yiming Wu${ }^{1}$, Hanxu Wang ${ }^{1}$, Shan Huang ${ }^{2}$ and Guohua Wang ${ }^{1,3^{*}}$
}

\begin{abstract}
Background: Various methods for differential expression analysis have been widely used to identify features which best distinguish between different categories of samples. Multiple hypothesis testing may leave out explanatory features, each of which may be composed of individually insignificant variables. Multivariate hypothesis testing holds a non-mainstream position, considering the large computation overhead of large-scale matrix operation. Random forest provides a classification strategy for calculation of variable importance. However, it may be unsuitable for different distributions of samples.

Results: Based on the thought of using an ensemble classifier, we develop a feature selection tool for $\underline{\mathbf{d}}$ differential expression analysis on expression profiles (i.e., ECFS-DEA for short). Considering the differences in sample distribution, a graphical user interface is designed to allow the selection of different base classifiers. Inspired by random forest, a common measure which is applicable to any base classifier is proposed for calculation of variable importance. After an interactive selection of a feature on sorted individual variables, a projection heatmap is presented using $\mathrm{k}$-means clustering. ROC curve is also provided, both of which can intuitively demonstrate the effectiveness of the selected feature.

Conclusions: Feature selection through ensemble classifiers helps to select important variables and thus is applicable for different sample distributions. Experiments on simulation and realistic data demonstrate the effectiveness of ECFS-DEA for differential expression analysis on expression profiles. The software is available at http://bio-nefu.com/resource/ecfs-dea.
\end{abstract}

Keywords: Feature selection, Classification, Accumulation, Expression profiles, Differential expression analysis

\section{Background}

Differential expression analysis (DEA) is widely adopted to identify a feature for best characterizing the expression difference between groups of individuals (e.g., healthy ones and those affected with a disease) [1]. Multiple hypothesis testing, which evaluates more than one hypothesis simultaneously, plays an important role in DEA. Corresponding tools such as SAM [2], limma [3],

\footnotetext{
*Correspondence: ghwang@nefu.edu.cn

tThe authors wish it to be known that, in their opinion, the first three authors should be regarded as Joint First Authors.

${ }^{1}$ College of Information and Computer Engineering, Northeast Forestry University, No.26 Hexing Road, 150040 Harbin, China

${ }^{3}$ State Key Laboratory of Tree Genetics and Breeding, Northeast Forestry University, No.26 Hexing Road, 150040 Harbin, China

Full list of author information is available at the end of the article
}

multtest [4], etc. have been produced for detecting differentially expressed variables. As a matter of fact, multiple hypothesis testing may leave out an explanatory signature. A selected feature expressed differently may not be composed of individually significant variables [5]. Although multivariate hypothesis testing may choose a proper feature, it still holds a non-mainstream position [6], considering the need for a large computation overhead of large-scale matrix operation.

Unlike statistical hypothesis testing, classification-based feature selection concentrates on better classification results of a certain subspace in many aspects such as sequence analysis $[7,8]$, site identification [9-12], protein classification $[13,14]$, protein identification $[15,16]$,

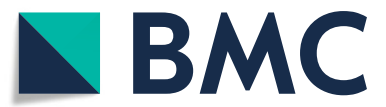

(C) The Author(s). 2020 Open Access This article is distributed under the terms of the Creative Commons Attribution 4.0 International License (http://creativecommons.org/licenses/by/4.0/), which permits unrestricted use, distribution, and reproduction in any medium, provided you give appropriate credit to the original author(s) and the source, provide a link to the Creative Commons license, and indicate if changes were made. The Creative Commons Public Domain Dedication waiver (http://creativecommons.org/publicdomain/zero/1.0/) applies to the data made available in this article, unless otherwise stated. 
protein fold recognition [17-19], protease substrate prediction $[20,21]$ and protein backbone torsion angle prediction [22]. Thus, predictive variables [23-25] are selected according to classification results of a certain classifier. Random forest [26, 27] is a case in point. It utilizes decision trees as the base classifier, which may be unsuitable for different distributions of samples. We have developed JCD-DEA [28], which is a feature selection tool combining hypothesis testing with classification strategy. However, JCD-DEA employs a bottom-up feature enumeration strategy, which is time consuming.

In this paper, we develop a top-down classificationbased feature selection tool, i.e. ECFS-DEA, for differential expression analysis. In addition to random forest (RF), one of the other three classifiers, i.e., Fisher's linear discriminant analysis (LDA), k-nearest-neighbor $(\mathrm{kNN})$ and support vector machine (SVM), can be interactively selected to be the base classifier in accordance with different sample distributions. Under the development environment of Python 3.5, ECFS-DEA applicable to various execution environments such as a personal computer, a workstation or a large-scale cluster in Windows, Linux or Mac, can be used to identify the feature which best distinguishes between different categories of samples on expression profiles such as RNA-seq data, microarrays, etc.

\section{Method}

ECFS-DEA offers two main functions, i.e. feature selection and feature validation. Feature selection part contains five steps, as illustrated in Fig. 1. Firstly, the category of the base classifier is to be interactively appointed. RF, LDA, kNN and SVM are the alternative base classifier. The base classifier number $r$ is also to be set. Meanwhile, the path of the input file, the data format and the execution environment are to be selected. Secondly, samples are randomly divided into training and testing groups in balance. Thirdly, a resampling procedure is constructed for the accumulation of variable importance. The resampling round is equivalent to the number of the base classifiers. In each round $j, 70 \%$ of training samples are randomly selected in the entire feature space

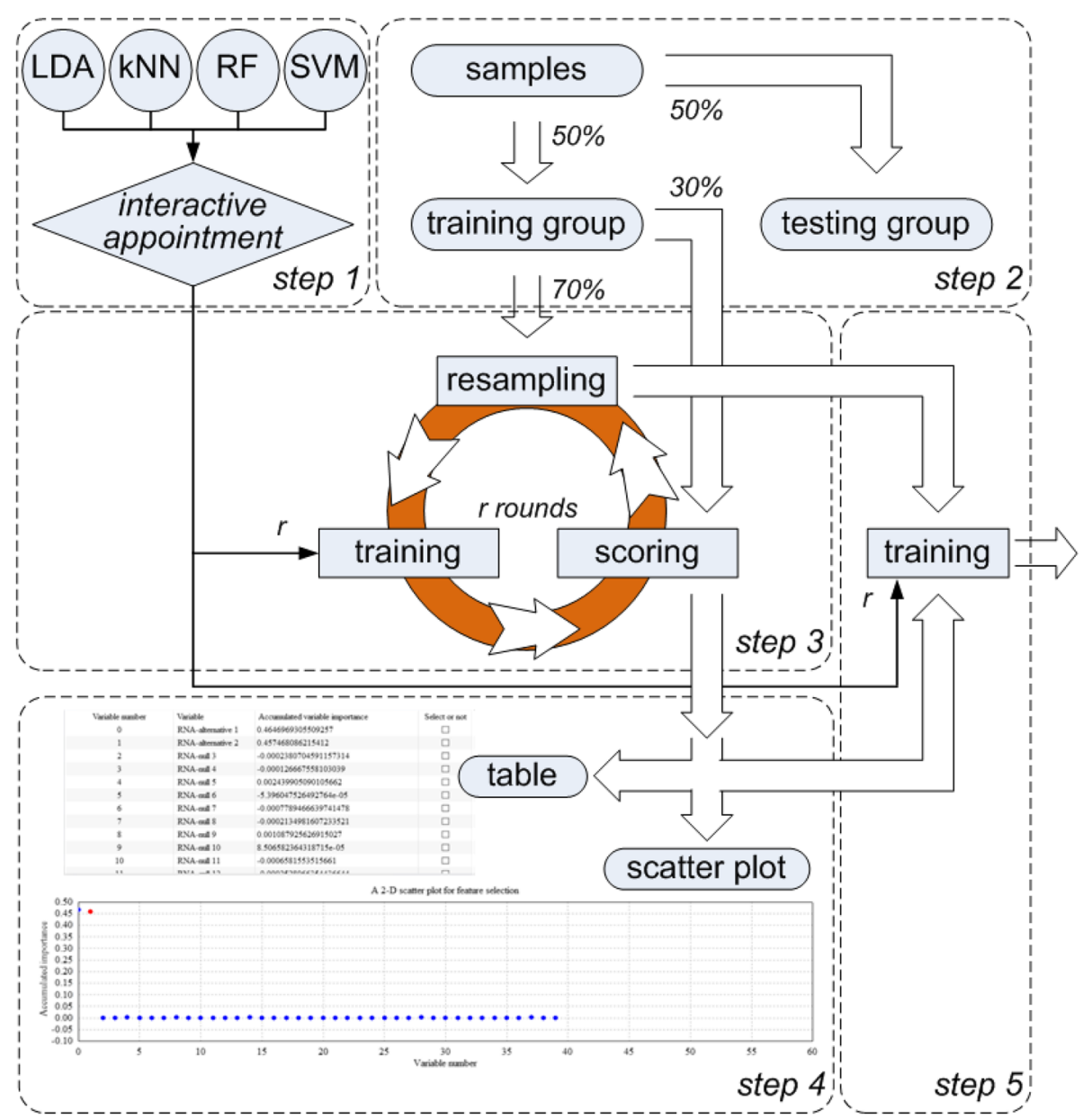

Fig. 1 Schematic of feature selection part in ECFS-DEA 
for training each classifier; while, the remaining $30 \%$ of training samples are the out-of-bag data for calculating the classification error rate $E r r$. As to each variable $i$, only one time permutation of its expression levels on the out-of-bag data is made, and the corresponding classification error rate is presented as $\operatorname{Err}_{j}^{0}(i)$. After $r$ rounds of resampling, the importance of variable $i$ is achieved as $\sum_{j=1}^{n}\left(E r r_{j}^{0}(i)-E r r_{j}\right) / r$. Fourthly, a feature can be manually selected in a table with the individual variables sorted in descending order according to achieved variable importance or in a 2-D scatter plot with its horizontal and vertical coordinates corresponding to the variable indices and the accumulated importance, respectively. Fifthly, an ensemble classifier composed of $r$ same base classifiers is to be trained using the expression levels of the training samples on the selected feature.

As to feature validation part, the testing samples are needed. Aiming at the expression levels of the testing set on the selected feature, a scatter plot in 1-D, 2-D or 3-D subspace can be illustrated. The corresponding ROC curve is also provided. Besides, a projection heatmap which displays discrete projection values (i.e., classification results) from the expression levels of the selected feature, is presented. Using the trained classifier, the classification results of the testing set on the selected feature are reordered based on k-means clustering. Accompanied with the expression levels and the labels, the reordered classification results are shown in the projection heatmap.

\section{Implementation}

ECFS-DEA is written mainly in Python 3.5, distributed under GNU GPLv3. Considering the existence of repeating steps in ECFS-DEA, we make a two-step implementation: a client part in Client.zip for executing
GUI, and a server part in Server.zip which is designed to run on the cluster server that using Portable Batch System(PBS) as scheduling program. The client part also contains codes for analyzing expression profiles, if ECFS-DEA can only run on a personal computer or a workstation.

The parameter setting step of feature selection part is illustrated in Fig. 2. The file path, data format, execution environment, etc. are set. Besides, the category of the base classifier is interactively assigned. The number of the base classifier which is also the resampling round needs to be appointed. Sample splitting is performed after parameter setting. Once the accumulation of variable importance is fulfilled, the obtained scores can be listed in a table or a scatter plot form for manual selection, as illustrated in Figs. 3 and 4 respectively.

In a table form as shown in Fig. 3, one can click the checkbox of the fourth column called "select or not" for fulfilling feature selection. The third column header can be clicked to rank. In a scatter plot form as shown in Fig. 4, one can double click the scatter to select the variable to be a part of a feature with its color changed red and vice versa. When users move the mouse around the scatter, the variable information can be displayed.

Figures 5, 6 and 7 together illustrate the panel for feature validation part of ECFS-DEA in Windows. Corresponding panels in Linux or Mac are almost the same. After pressing button "Scatter plot", a 1-D, 2-D or 3-D scatter plot of the selected feature is shown in Fig. 5. Scatter plots with different colors denote samples from different groups. After pressing button "ROC curve", the ROC curve of the selected feature is provided, as shown in Fig. 6. After pressing button "Projection heatmp", the projection heatmap of the selected feature is presented, as shown in Fig. 7. A discrete projection from the expression

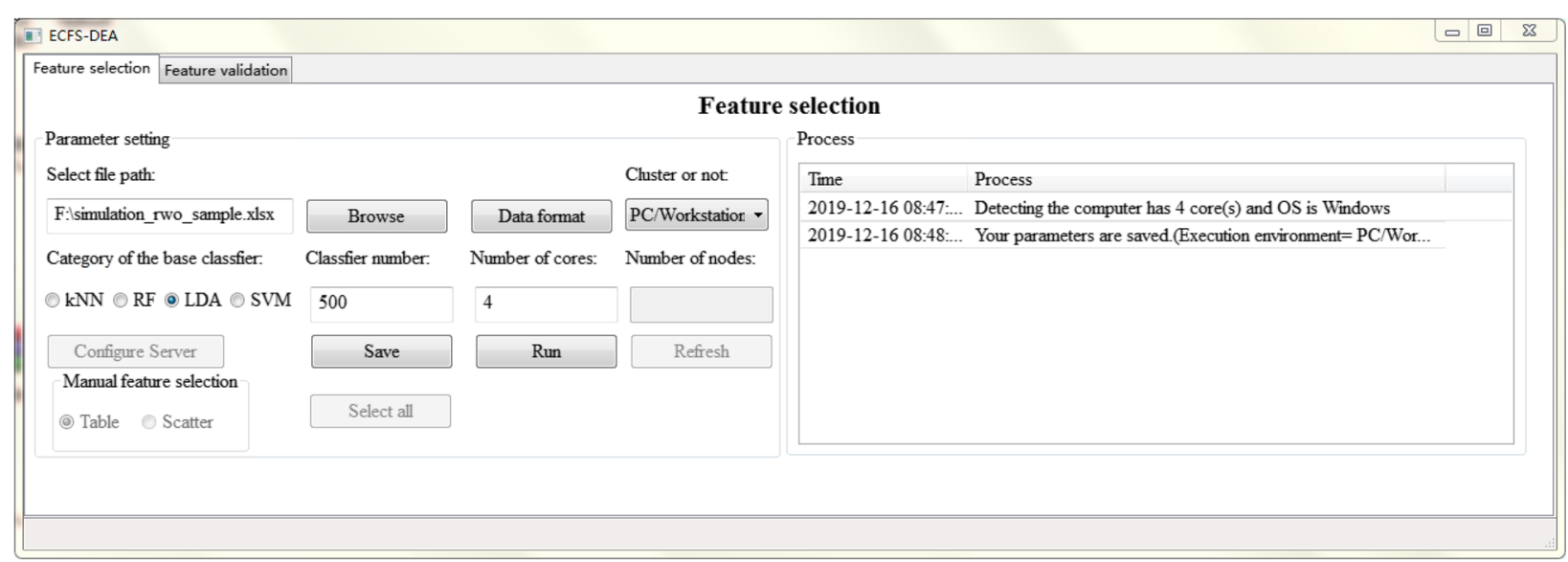

Fig. 2 The parameter setting step of feature selection part in ECFS-DEA 


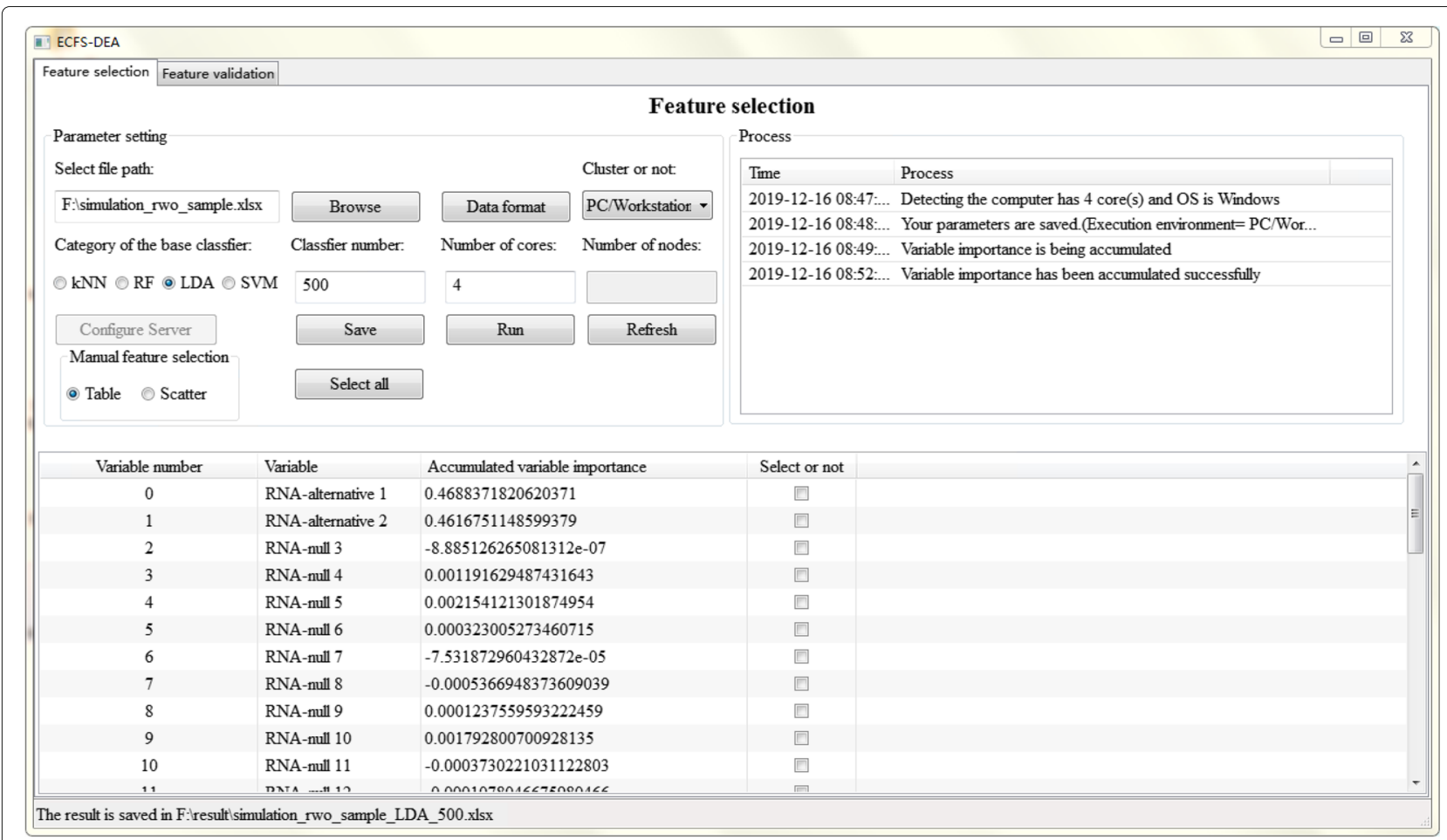

Fig. 3 Feature selection step using a table form in ECFS-DEA

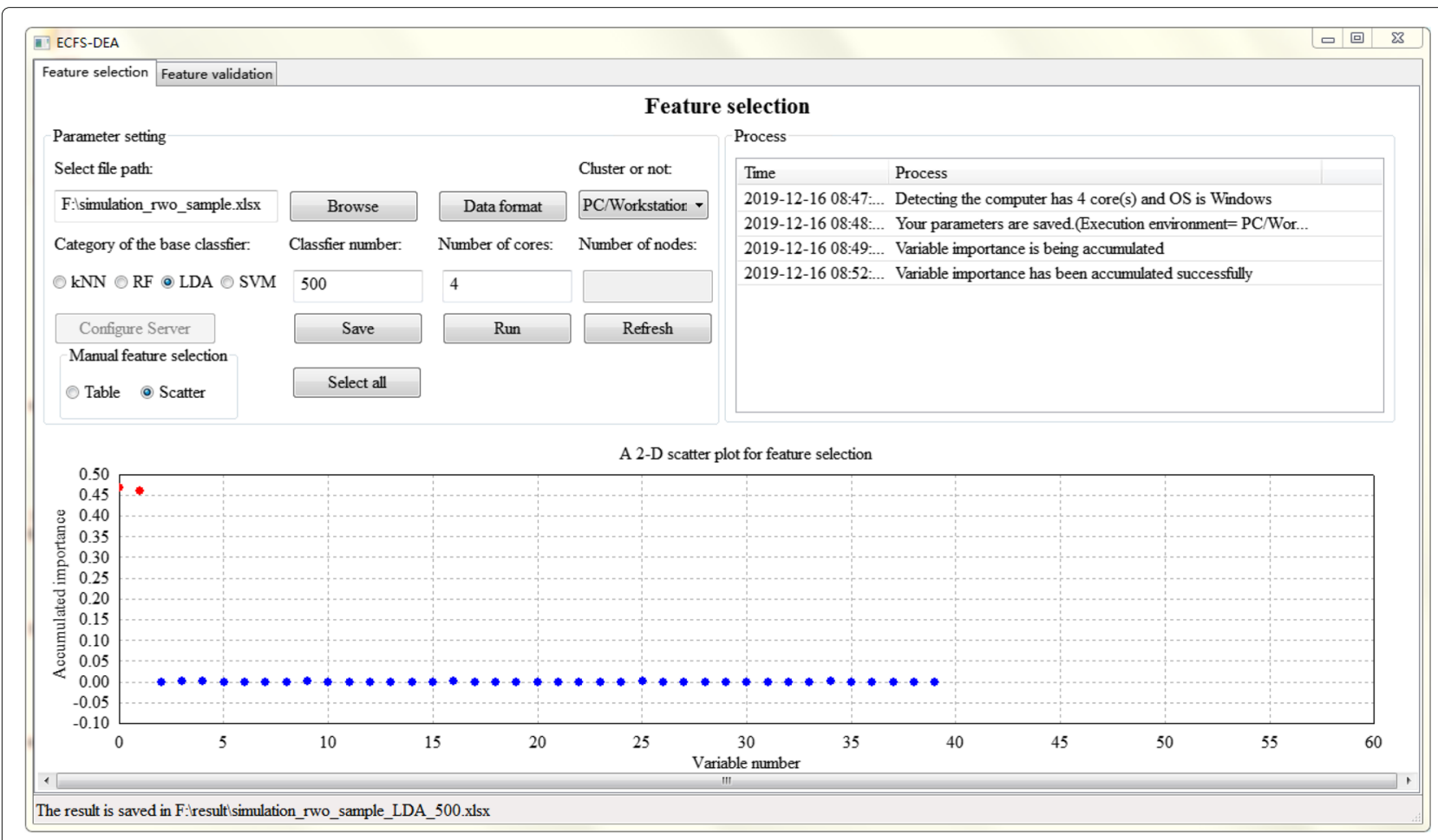

Fig. 4 Feature selection step using a scatter plot in ECFS-DEA 


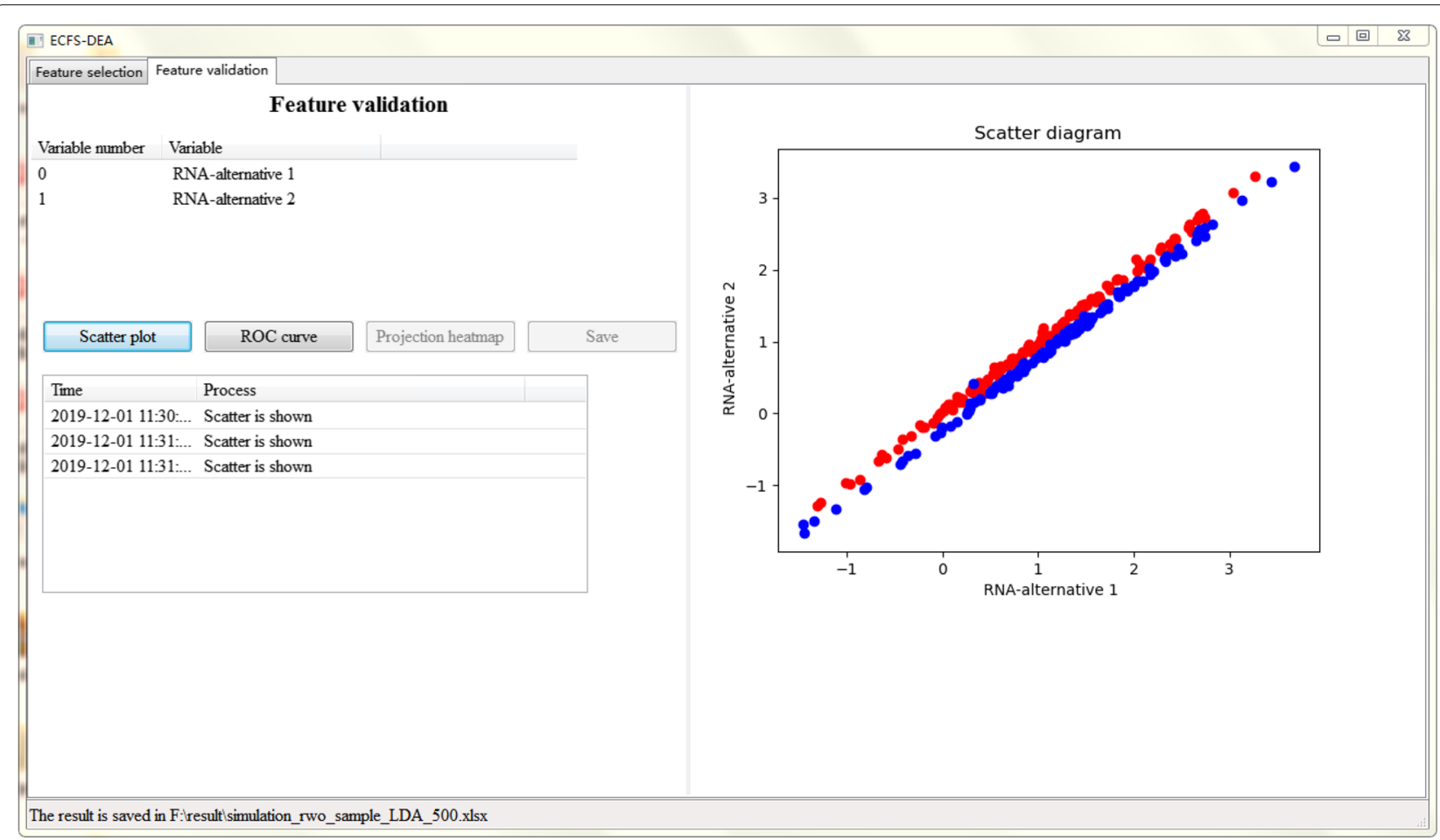

Fig. 5 Feature validation step using a scatter plot in ECFS-DEA

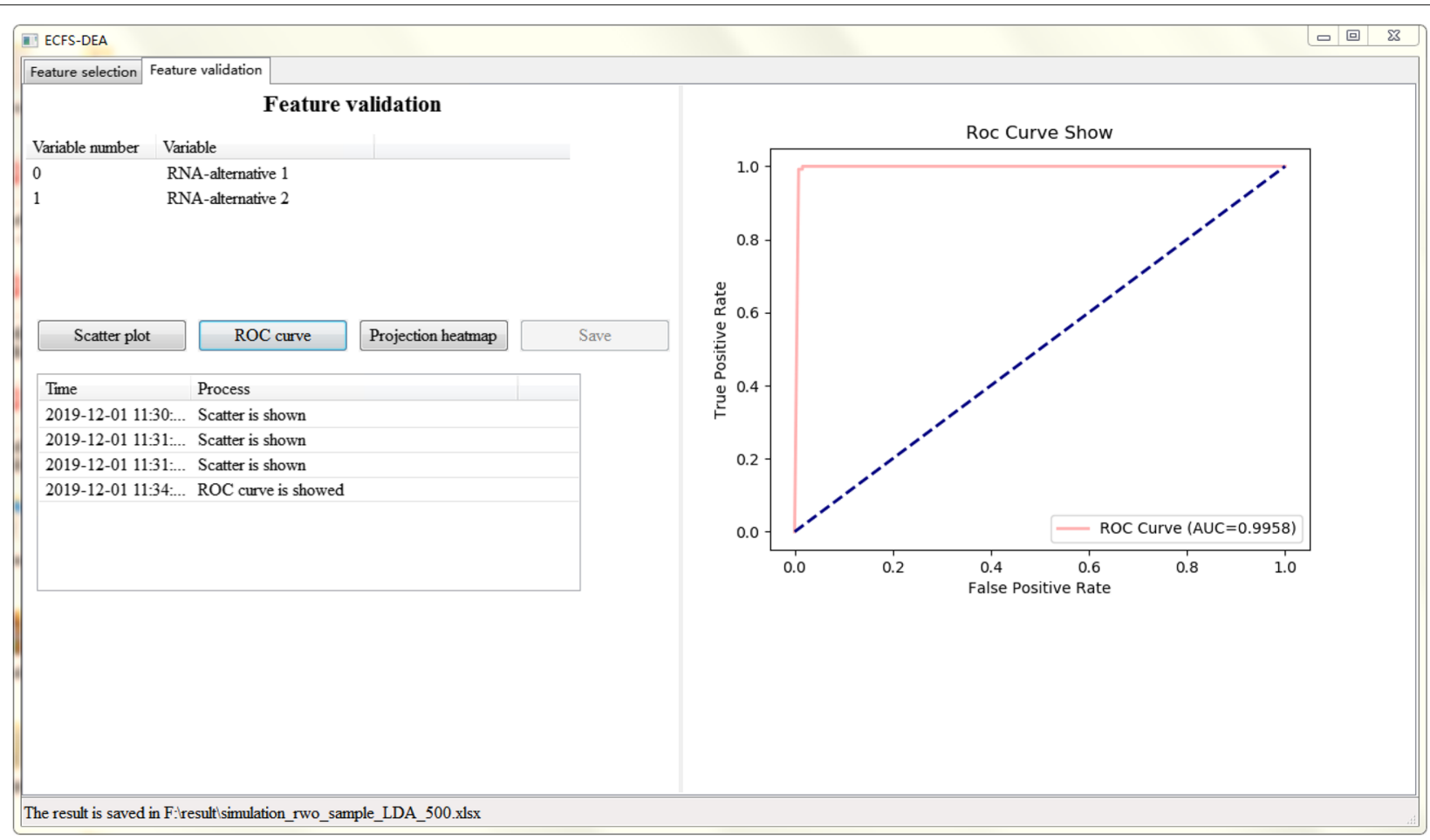

Fig. 6 Feature validation step using a ROC curve in ECFS-DEA 


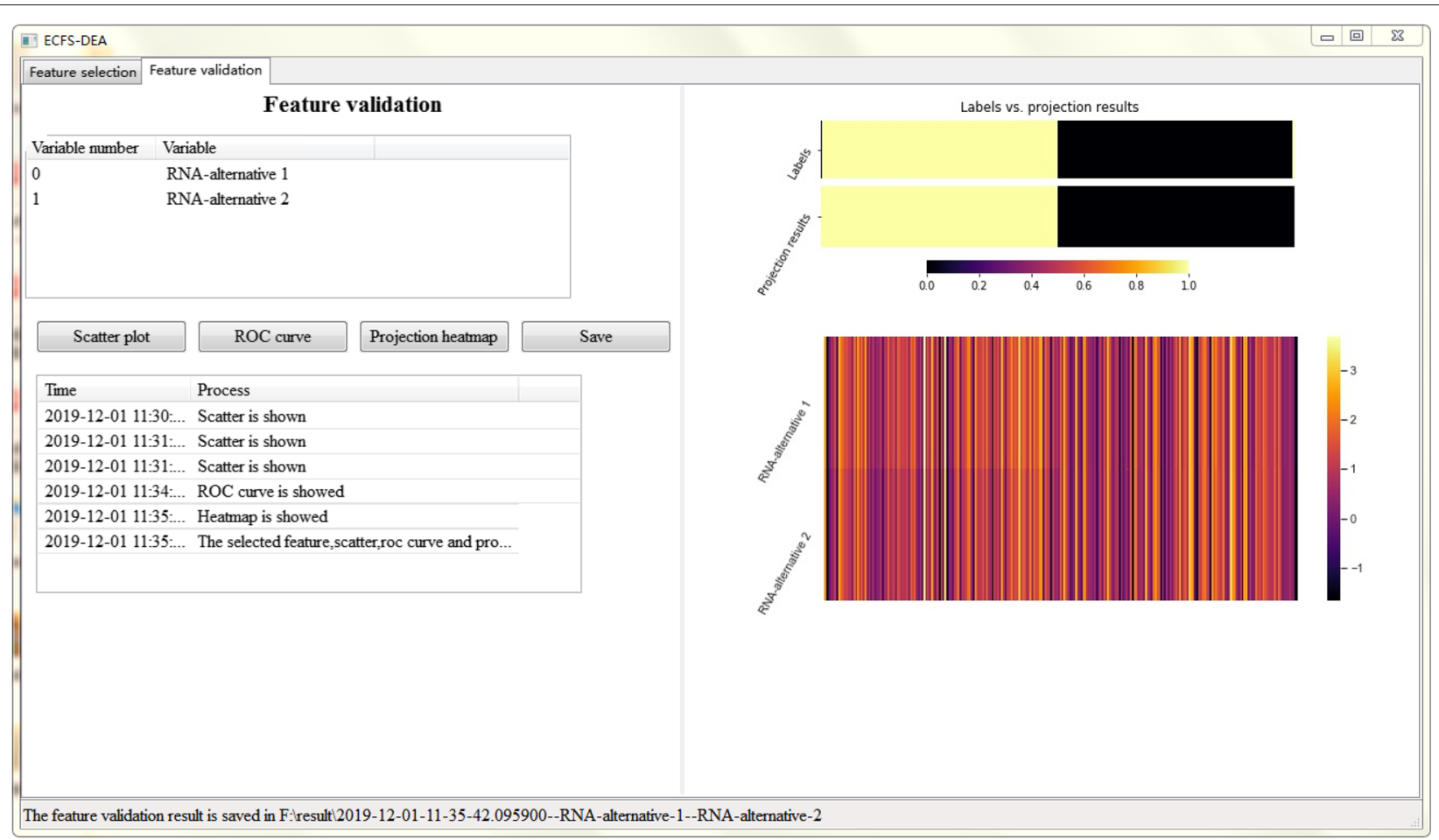

Fig. 7 Feature validation step using a projection heatmap in ECFS-DEA

levels of the selected feature (i.e., the classification results) is made. Samples are reordered according to the $\mathrm{k}$-means clustering results of the projection values.

Detailed software documentation and tutorial are presented on http://bio-nefu.com/resource/ecfs-dea.

\section{Results}

\section{Feature selection on the simulated data}

In order to demonstrate the effectiveness of our ECFSDEA, a simulated data consisting of 250 positive and 250 negative samples in a 40 dimensional space is constructed.

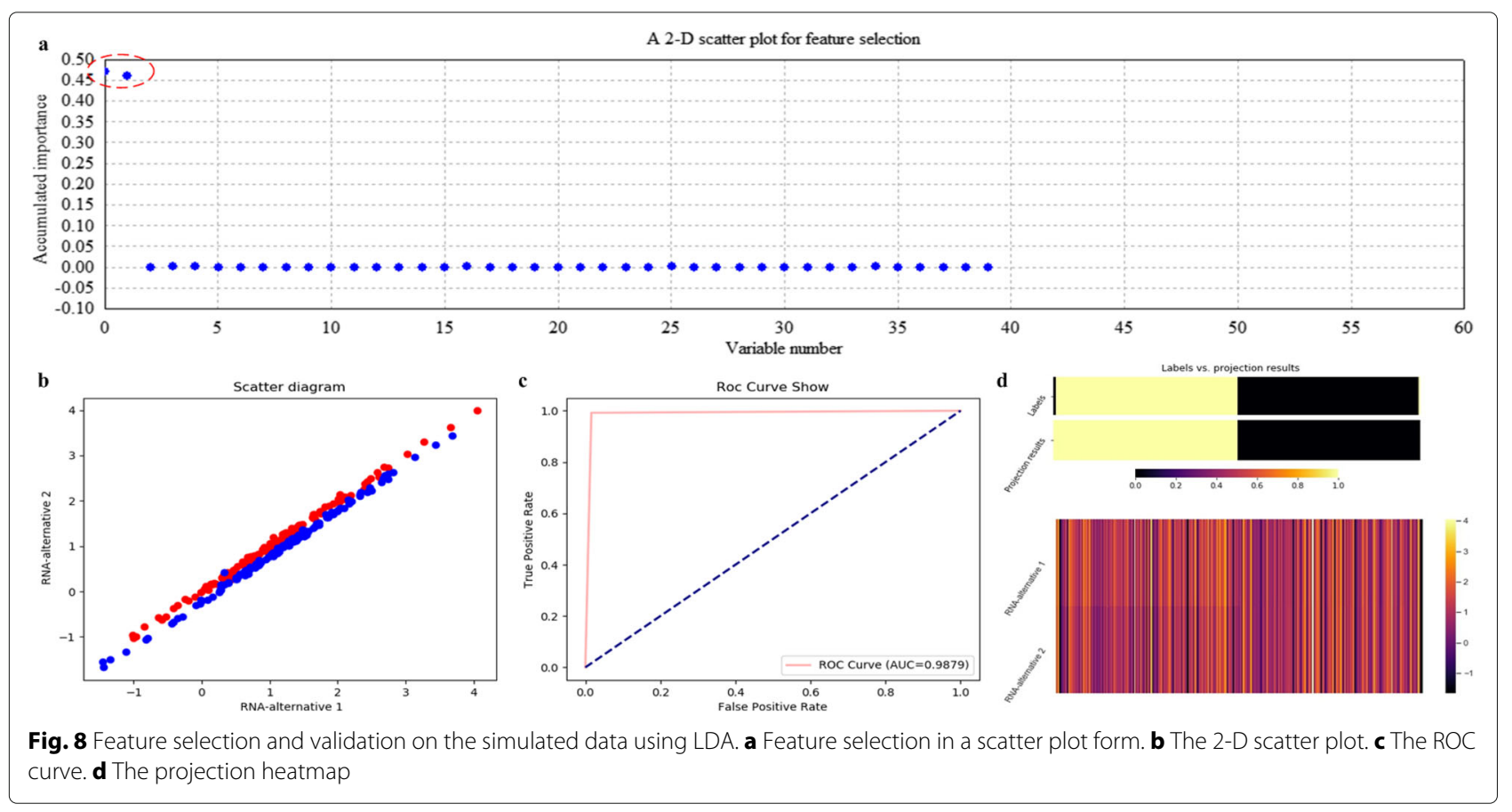



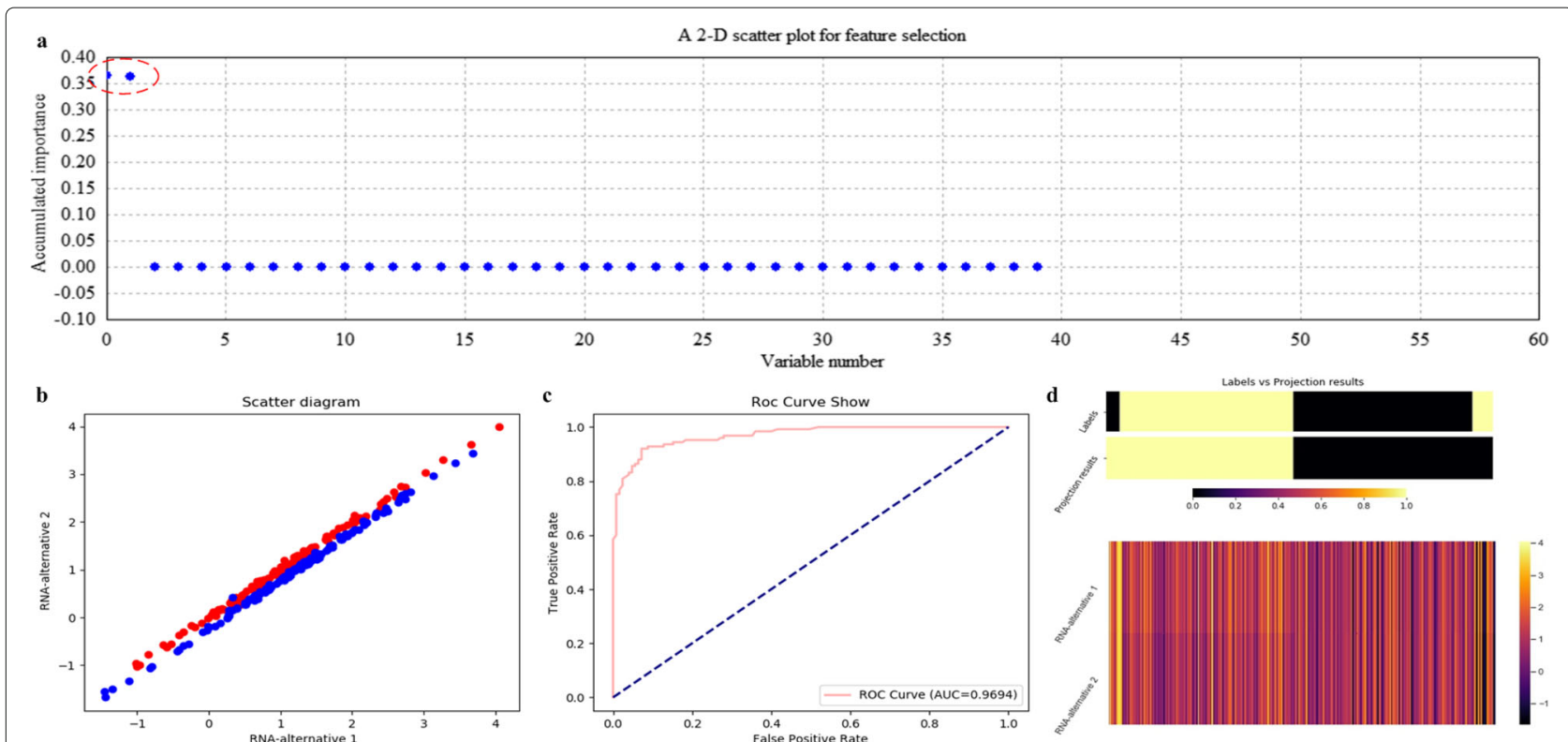

Fig. 9 Feature selection and validation on the simulated data using kNN ( $k=5)$. a Feature selection in a scatter plot form. $\mathbf{b}$ The 2-D scatter plot. $\mathbf{c}$ The ROC curve. d The projection heatmap

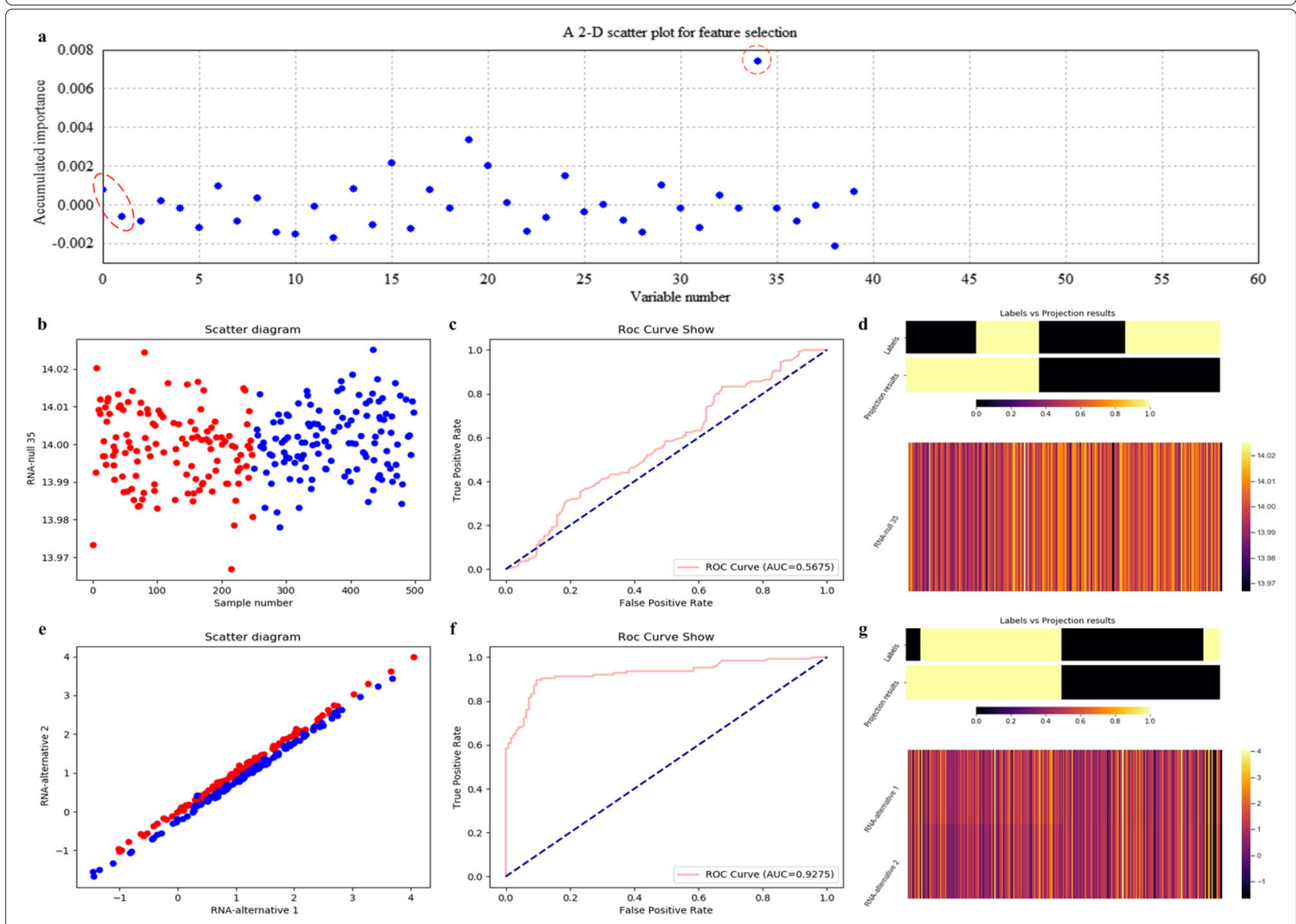

Fig. 10 Feature selection and validation on the simulated data using RF. a Feature selection in a scatter plot form. $\mathbf{b}$ The 1-D scatter plot of the selected feature with $x$ and $y$ coordinates to be sample indices and expression values. c The ROC curve of the selected feature. $\mathbf{d}$ The projection heatmap of the selected feature. $\mathbf{e}$ The 2-D scatter plot of the significant pair. $\mathbf{f}$ The ROC curve of the significant pair. $\mathbf{g}$ The projection heatmap of the significant pair 
38 variables of them follow 38 normal distributions, each of which is independently and identically distributed and keeps a random mean value in range from 10 to 30 and a common standard deviation 0.01 . The additional variable pair, i.e., miRNA-alternative 1 and miRNA-alternative 2, follows a bivariate normal distribution and has a clear category distinction. The mean vectors corresponding to positive and negative samples are $(1,1)^{T}$ and $(1.11,0.89)^{T}$, respectively. Correspondingly, a same covariance matrix, which is expressed as $\left(\begin{array}{cc}1 & 0.999 \\ 0.999 & 1\end{array}\right)$, is kept.

We made this simulated data in order to show the effectiveness of using LDA compared to RF. Considering the comparability with real data, we made the sample size to be 500. This data can be downloaded at http://bio-nefu. com/resource/ecfs-dea.

Using ECFS-DEA with LDA assigned as the base classifier, the significant variable pair is properly selected on the training set according to the accumulation of variable importance after 500 rounds of resampling, as shown in Fig. 8a. Meanwhile, the corresponding 2-D scatter plot, the ROC curve and the projection heatmap of the testing group are illustrated in turn, as shown in Fig. 8b, c and d. It can be seen in Fig. 8b that the testing set is 2-D but not 1-D linearly separable. The corresponding ROC curve is shown in Fig. 8c. As to Fig. 8d, a discrete projection from the expression levels of the selected variable pair (i.e., the classification results) is made. Samples are reordered according to the k-means cluster results of the projection values. It can be seen in Fig. $8 \mathrm{~d}$ that a sample labeled 0 is misclassified, which corresponds to the blue point within the points labeled red in Fig. 8b.

Figure 9 illustrates the variable selection results using kNN $(k=5)$ on the simulated data after 500 rounds of resampling. In Fig. 9a, miRNA-alternative 1 and miRNAalternative 2 are also intuitively selected. Correspondingly, the scatter plot, the ROC curve and the projection heatmap are listed in Fig. 9b, c and d, which show the

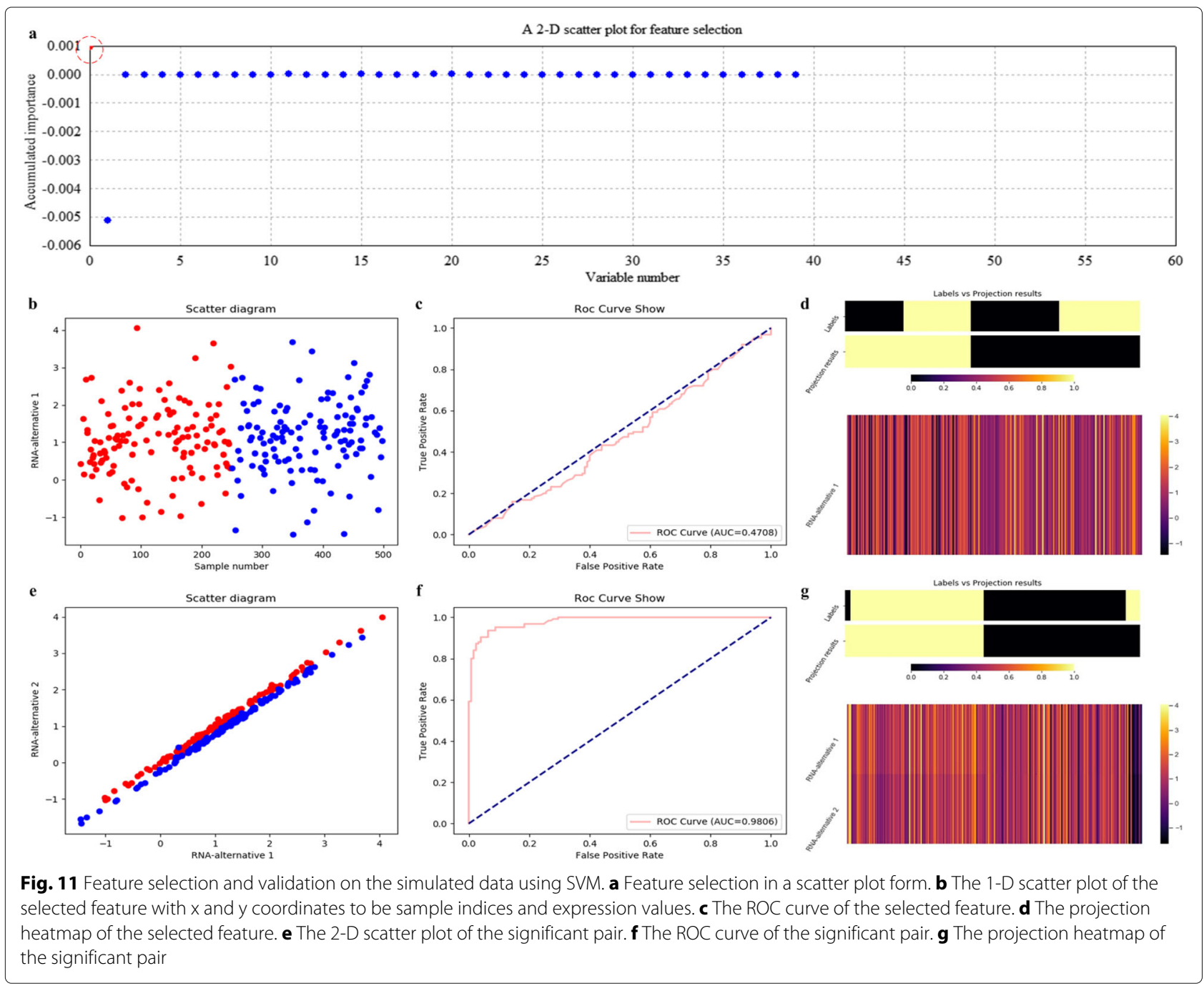


effectiveness of choosing $\mathrm{kNN}$ as the base classifier on the simulated data.

Figure 10 illustrates the variable selection results using RF on the simulated data after 500 rounds of resampling. As shown Fig. 10a, it is miRNA-null 35 but not miRNA-alternative 1 and miRNA-alternative 2 that is selected. And it is considered as a false selection. This directly demonstrates that RF is not applicable to any data with different sample distributions. Correspondingly, the scatter plot, the ROC curve and the projection heatmap of miRNA-null 35 are listed in Fig. 10b, c and $\mathrm{d}$. All these results further demonstrate the above phenomenon.

Figure 10b illustrates a 1-D scatter plot of the selected miRNA-null 35 using RF. The horizontal and vertical coordinates correspond to sample indices and expression levels, respectively. It can be seen that samples from two categories of the testing data are indivisible according to the vertical coordinate values. Figure 10c illustrates a poor ROC curve. As to Fig. 10d, it can be seen that the two clusters derived from the projection results contain many wrong labels.

Correspondingly, we also make the scatter plot, the ROC curve and the projection heatmap using RF on miRNA-alternative 1 and miRNA-alternative 2 , which are listed in Fig. 10e, f and g, respectively. The experimental results of RF have improved; however, its ROC curve and projection heatmap are inferior to those of kNN and LDA.

As to SVM which is assigned as the base classifier, it is only miRNA-alternative 1 but not the significant pair that is selected, as illustrated in Fig. 11a. It indicates that SVM is not applicable to the simulated data for feature selection. Correspondingly, the scatter plot, the ROC curve and the projection heatmap of miRNAalternative 1 are listed in Fig. 11b, c and d. On the contrary, we also make the scatter plot, the ROC curve and the projection heatmap using SVM on miRNAalternative 1 and miRNA-alternative 2 , as shown in Fig. $11 \mathrm{e}, \mathrm{f}$ and $\mathrm{g}$.

The quantitative results on the simulated data with measures such as confusion matrix, precision, recall and F1-measure are listed in Table 1. In fact, it can be seen that RF and SVM achieve poor results, for they correspond to lower scores of accumulated importance compared with those of LDA and kNN, as shown in Figs. 8a, 9a, 10a and 11a, respectively. All the experimental results indicate that LDA is a more appropriate classifier for feature selection on the simulated data.

\section{Feature selection on GSE22058}

We also performed experiments on GSE22058 [29] which is a public dataset containing 96 samples associated with liver tumor and 96 samples corresponded to adjacent liver

Table 1 Quantitative results on the simulation data

\begin{tabular}{|c|c|c|c|c|c|c|c|c|}
\hline \multirow{2}{*}{$\frac{\text { Base classifier }}{\text { LDA }}$} & \multirow{2}{*}{$\begin{array}{l}\text { Variable number } \\
{[0,1]^{T}}\end{array}$} & \multicolumn{3}{|c|}{ Confusion matrix } & \multirow{2}{*}{$\begin{array}{l}\text { Positive class } \\
\mathrm{a}\end{array}$} & \multirow{2}{*}{$\begin{array}{l}\text { Precision } \\
0.992\end{array}$} & \multirow{2}{*}{$\begin{array}{l}\text { Recall } \\
0.984\end{array}$} & \multirow{2}{*}{$\frac{\text { F1-measure }}{0.988}$} \\
\hline & & classified as & a & $\mathrm{b}$ & & & & \\
\hline & & label a & 123 & 2 & $b$ & 0.984 & 0.992 & 0.988 \\
\hline & & label b & 1 & 124 & weighted average & 0.988 & 0.988 & 0.988 \\
\hline \multirow[t]{3}{*}{ kNN } & {$[0,1]^{T}$} & classified as & a & $b$ & a & 0.906 & 0.928 & 0.917 \\
\hline & & label a & 116 & 9 & $b$ & 0.926 & 0.904 & 0.915 \\
\hline & & label b & 12 & 113 & weighted average & 0.916 & 0.916 & 0.916 \\
\hline \multirow[t]{6}{*}{ RF } & 34 & classified as & a & $b$ & a & 0.528 & 0.448 & 0.485 \\
\hline & & label a & 56 & 69 & $b$ & 0.521 & 0.600 & 0.558 \\
\hline & & label b & 50 & 75 & weighted average & 0.524 & 0.524 & 0.522 \\
\hline & {$[0,1]^{T}$} & classified as & a & $b$ & a & 0.897 & 0.904 & 0.900 \\
\hline & & label a & 113 & 12 & $b$ & 0.903 & 0.896 & 0.899 \\
\hline & & label b & 13 & 112 & weighted average & 0.900 & 0.900 & 0.899 \\
\hline \multirow[t]{6}{*}{ SVM } & 0 & classified as & a & $\mathrm{b}$ & a & 0.467 & 0.400 & 0.431 \\
\hline & & label a & 50 & 75 & $b$ & 0.476 & 0.544 & 0.508 \\
\hline & & label b & 57 & 68 & weighted average & 0.472 & 0.472 & 0.470 \\
\hline & {$[0,1]^{T}$} & classified as & a & $b$ & a & 0.909 & 0.960 & 0.934 \\
\hline & & label a & 120 & 5 & $b$ & 0.958 & 0.904 & 0.930 \\
\hline & & label b & 12 & 113 & weighted average & 0.933 & 0.932 & 0.932 \\
\hline
\end{tabular}




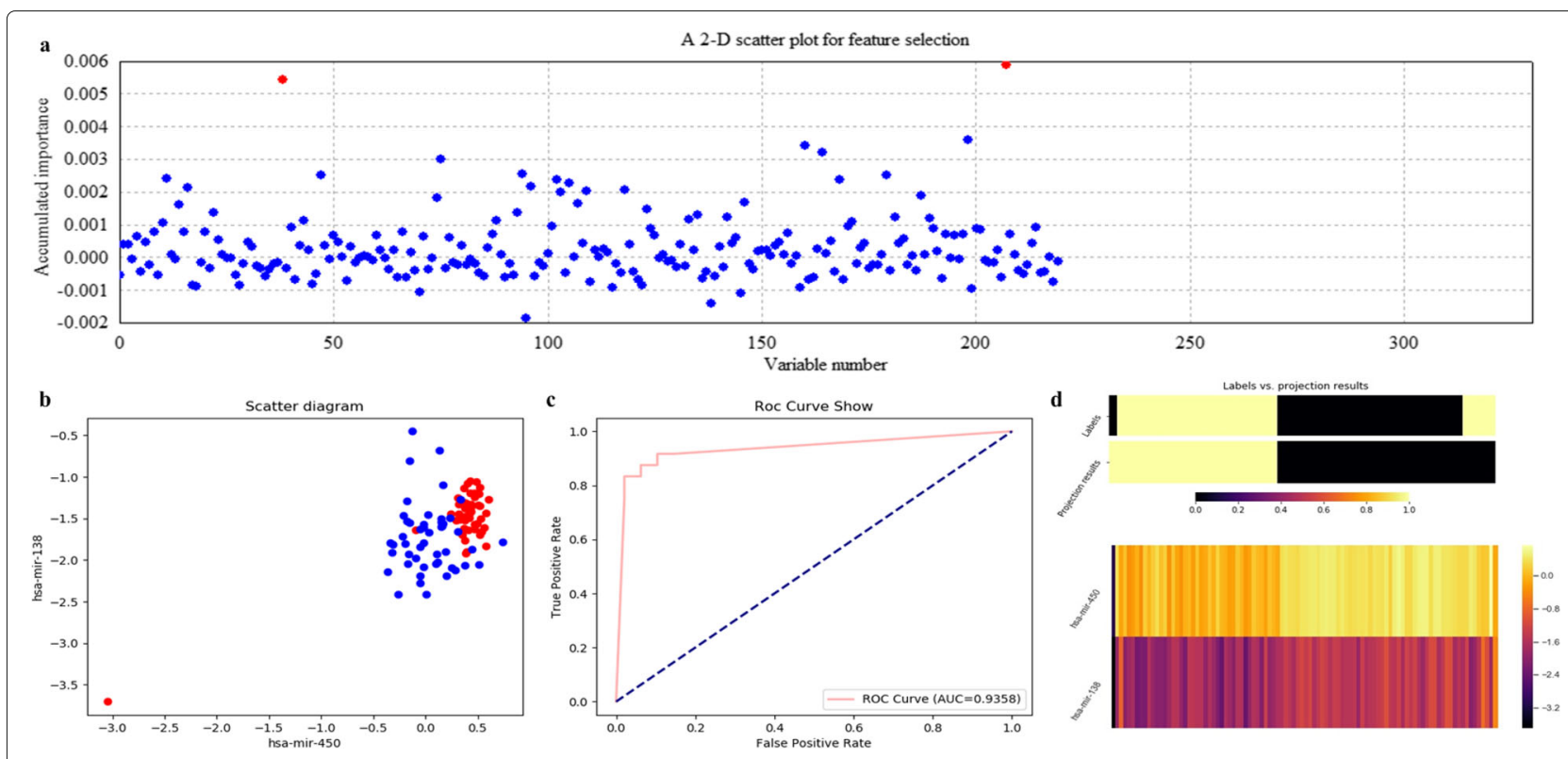

Fig. 12 Feature selection and validation on GSE22058 using LDA. a Feature selection in a scatter plot form. b The 2-D scatter plot. c The ROC curve. d The projection heatmap

non-tumor. In order to achieve a predictive feature from the 220 miRNAs, we utilized ECFS-DEA on GSE22058, with the base classifier to be LDA, kNN, RF and SVM.

Figures 12, 13, 14 and 15 illustrate qualitative results for feature selection using LDA, kNN (k=5), RF and SVM on GSE22058 after 500 rounds of resampling, respectively. In order to exhibit the scatter plots at the feature validation step, we restricted feature dimension less than four. Besides, quantitative results on GSE22058 with measures such as confusion matrix, precision, recall and F1-measure are listed in Table 2, with all possible variables intuitively selected. All the experimental results indicate that $R F$ is a more appropriate classifier to feature selection on GSE22058.

In addition, we searched the selected miRNAs using ECFS-DEA with RF to be the classifier, i.e., miR-188,

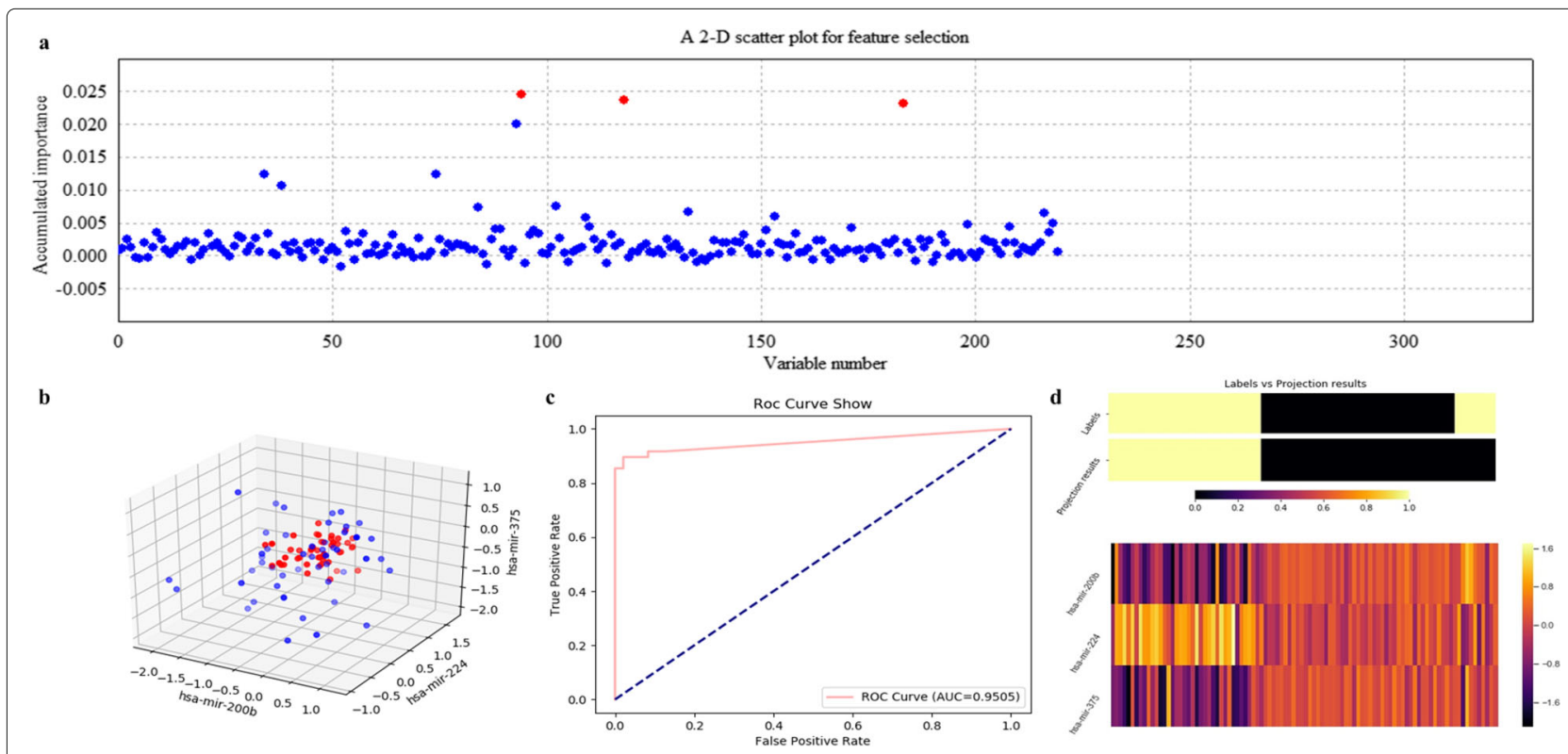

Fig. 13 Feature selection and validation on GSE22058 using kNN ( $k=5)$. a Feature selection in a scatter plot form. b The 3-D scatter plot. c The ROC curve. d The projection heatmap 


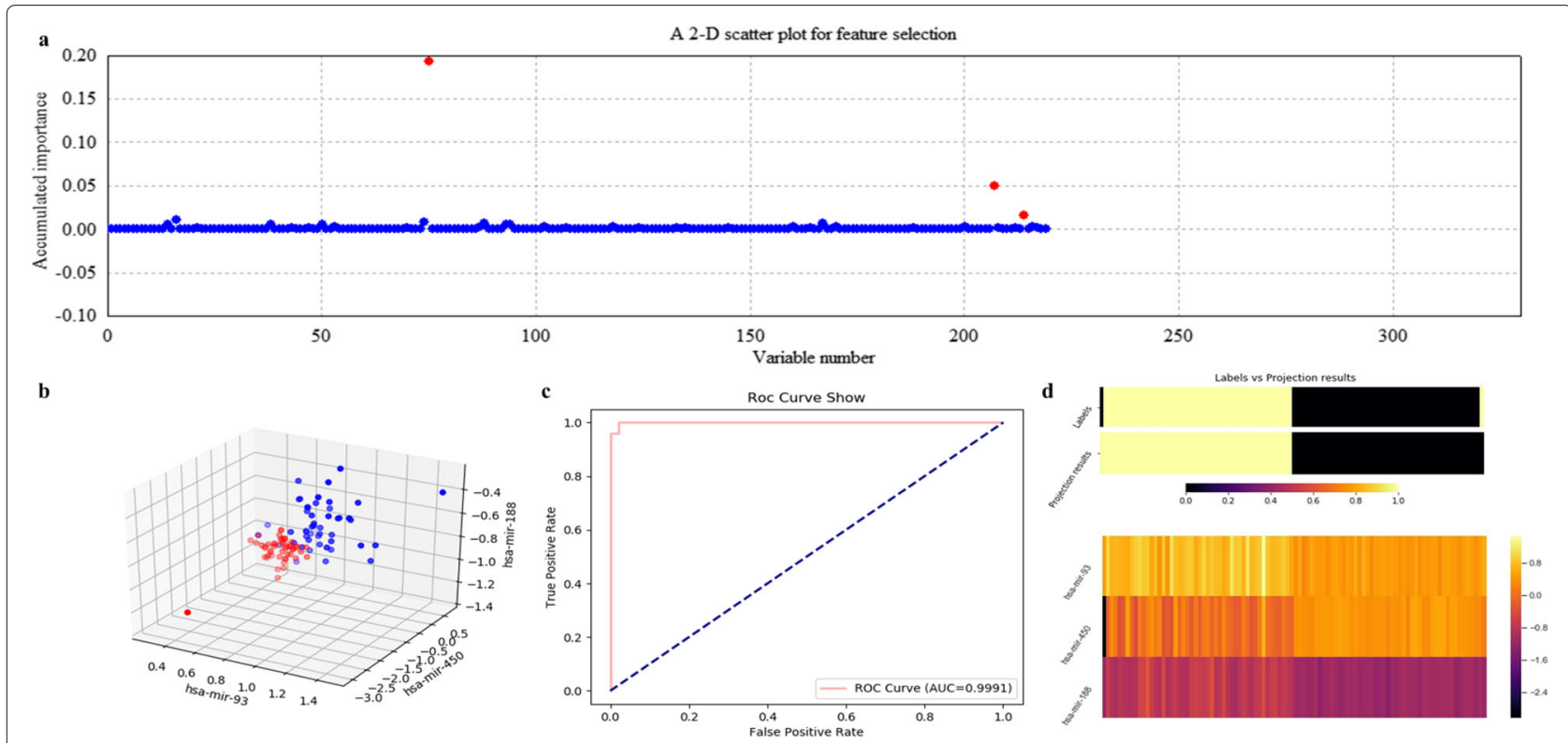

Fig. 14 Feature selection and validation on GSE22058 using RF. a Feature selection in a scatter plot form. b The 3-D scatter plot. c The ROC curve. d The projection heatmap

miR-450 and miR-93, on Web of Science with keywords to be such as liver tumor, hepatocellular carcinoma and HCC. Both miR-188 and miR-93 have been reported to be relevant to liver tumor. In fact, miR-188 achieved higher scores than other miRNAs, as shown in Fig. 14a. The retrieved results of miR-188 [30,31] have indirectly demonstrated the effectiveness of ECFS-DEA.

\section{Conclusions}

ECFS-DEA is a top-down classification-based tool for seeking predictive variables associated with different categories of samples on expression profiles. Other than prevailing differential expression analysis for class prediction, an ensemble classifier-based thought is proposed in this paper. According to accumulated scores of variable impor-

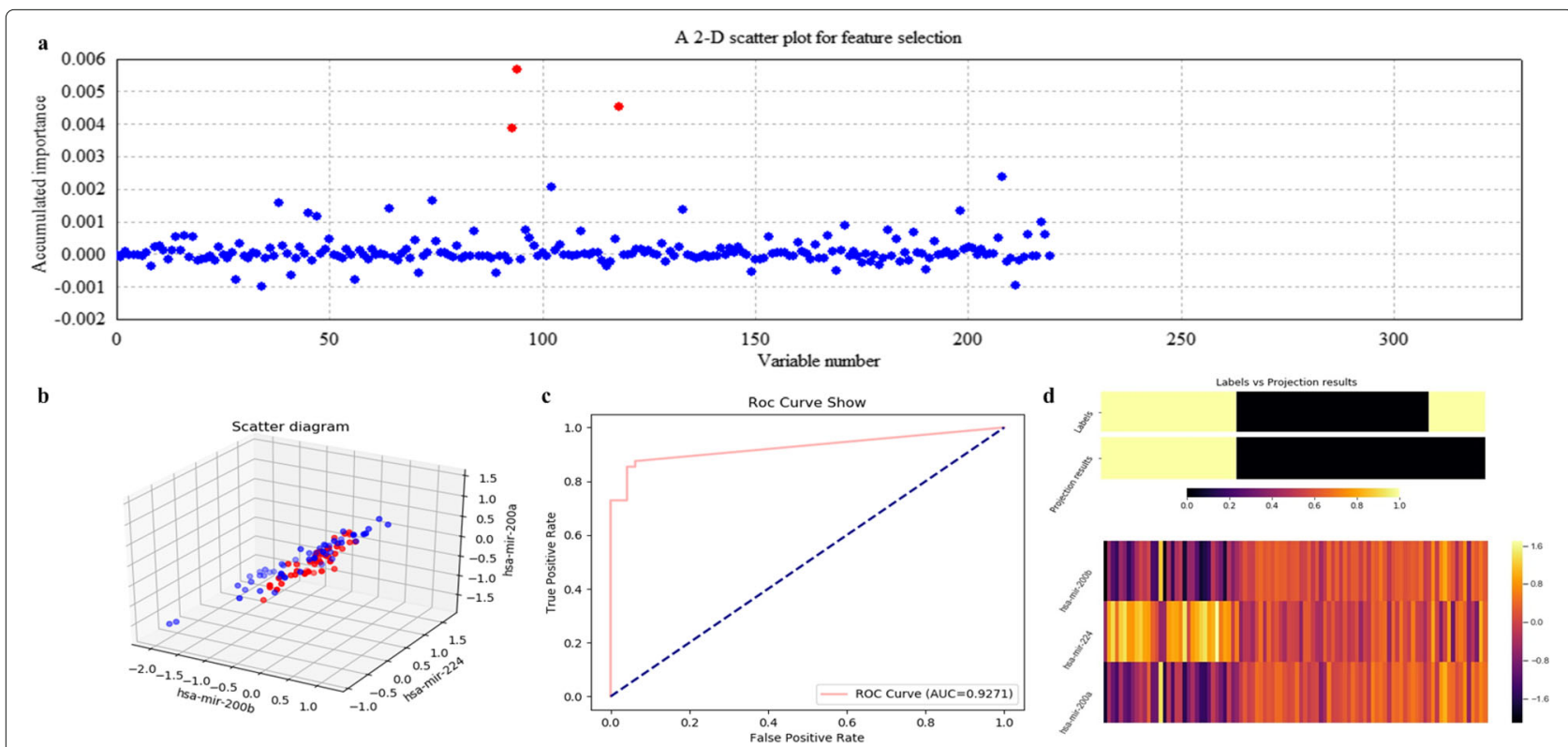

Fig. 15 Feature selection and validation on GSE22058 using SVM. a Feature selection in a scatter plot form. b The 3-D scatter plot. c The ROC curve. d The projection heatmap 
Table 2 Quantitative results on GSE22058

\begin{tabular}{|c|c|c|c|c|c|c|c|c|}
\hline \multirow{2}{*}{$\frac{\text { Base classifier }}{\text { LDA }}$} & \multirow{2}{*}{$\begin{array}{l}\text { Variable number } \\
207\end{array}$} & \multicolumn{3}{|c|}{ Confusion matrix } & \multirow{2}{*}{$\begin{array}{l}\text { Positive class } \\
\mathrm{a}\end{array}$} & \multirow{2}{*}{$\begin{array}{l}\text { Precision } \\
0.885\end{array}$} & \multirow{2}{*}{$\begin{array}{l}\text { Recall } \\
0.958\end{array}$} & \multirow{2}{*}{$\frac{\text { F1-measure }}{0.920}$} \\
\hline & & classified as & $\mathrm{a}$ & $\mathrm{b}$ & & & & \\
\hline & & label a & 46 & 2 & $\mathrm{~b}$ & 0.955 & 0.875 & 0.913 \\
\hline & & label b & 6 & 42 & weighted average & 0.920 & 0.916 & 0.917 \\
\hline & {$[207,38]^{\top}$} & classified as & a & $b$ & a & 0.852 & 0.958 & 0.902 \\
\hline & & label a & 46 & 2 & $b$ & 0.952 & 0.833 & 0.889 \\
\hline & & label b & 8 & 40 & weighted average & 0.902 & 0.895 & 0.895 \\
\hline & {$[207,38,198]^{T}$} & classified as & $\mathrm{a}$ & $b$ & $\mathrm{a}$ & 0.887 & 0.979 & 0.931 \\
\hline & & label a & 47 & 1 & $b$ & 0.977 & 0.875 & 0.923 \\
\hline & & label b & 6 & 42 & weighted average & 0.932 & 0.927 & 0.927 \\
\hline & {$[207,38,198,160]^{T}$} & classified as & a & $\mathrm{b}$ & a & 0.922 & 0.979 & 0.950 \\
\hline & & label a & 47 & 1 & $b$ & 0.978 & 0.917 & 0.947 \\
\hline & & label b & 4 & 44 & weighted average & 0.950 & 0.948 & 0.948 \\
\hline & {$[207,38,198$} & classified as & a & $b$ & a & 0.922 & 0.979 & 0.950 \\
\hline & & label a & 47 & 1 & $b$ & 0.978 & 0.917 & 0.947 \\
\hline & $160,164]^{\top}$ & label b & 4 & 44 & weighted average & 0.950 & 0.948 & 0.948 \\
\hline & {$[207,38,198$} & classified as & $\mathrm{a}$ & $b$ & a & 0.904 & 0.979 & 0.940 \\
\hline & & label a & 47 & 1 & $b$ & 0.977 & 0.896 & 0.935 \\
\hline & $160,164,75]^{\top}$ & label b & 5 & 43 & weighted average & 0.941 & 0.938 & 0.938 \\
\hline \multirow[t]{12}{*}{ kNN } & 94 & classified as & a & $b$ & a & 0.730 & 0.958 & 0.829 \\
\hline & & label a & 46 & 2 & $b$ & 0.939 & 0.646 & 0.765 \\
\hline & & label b & 17 & 31 & weighted average & 0.835 & 0.802 & 0.797 \\
\hline & {$[94,118]^{T}$} & classified as & $\mathrm{a}$ & $b$ & a & 0.800 & 1.000 & 0.889 \\
\hline & & label a & 48 & 0 & $b$ & 1.000 & 0.750 & 0.857 \\
\hline & & label b & 12 & 36 & weighted average & 0.900 & 0.875 & 0.873 \\
\hline & {$[94,118,183]^{\top}$} & classified as & a & $b$ & a & 0.828 & 1.000 & 0.906 \\
\hline & & label a & 48 & 0 & $b$ & 1.000 & 0.792 & 0.884 \\
\hline & & label b & 10 & 38 & weighted average & 0.914 & 0.896 & 0.895 \\
\hline & {$[94,118,183,93]^{\top}$} & classified as & $\mathrm{a}$ & $b$ & a & 0.787 & 1.000 & 0.881 \\
\hline & & label a & 48 & 0 & $b$ & 1.000 & 0.729 & 0.843 \\
\hline & & label b & 13 & 35 & weighted average & 0.893 & 0.865 & 0.862 \\
\hline \multirow[t]{12}{*}{ RF } & 75 & classified as & a & $b$ & $\mathrm{a}$ & 0.904 & 0.979 & 0.940 \\
\hline & & label a & 47 & 1 & $b$ & 0.977 & 0.896 & 0.935 \\
\hline & & label b & 5 & 43 & weighted average & 0.941 & 0.938 & 0.938 \\
\hline & {$[75,207]^{T}$} & classified as & $\mathrm{a}$ & $b$ & $a$ & 0.979 & 0.979 & 0.979 \\
\hline & & label a & 47 & 1 & $b$ & 0.979 & 0.979 & 0.979 \\
\hline & & label b & 1 & 47 & weighted average & 0.979 & 0.979 & 0.979 \\
\hline & {$[75,207,214]^{\top}$} & classified as & $a$ & $b$ & a & 0.979 & 0.979 & 0.979 \\
\hline & & label a & 47 & 1 & $b$ & 0.979 & 0.979 & 0.979 \\
\hline & & label b & 1 & 47 & weighted average & 0.979 & 0.979 & 0.979 \\
\hline & {$[75,207,214,16]^{\top}$} & classified as & a & $b$ & a & 0.980 & 1.000 & 0.990 \\
\hline & & label a & 48 & 0 & $b$ & 1.000 & 0.979 & 0.989 \\
\hline & & label b & 1 & 47 & weighted average & 0.990 & 0.990 & 0.990 \\
\hline \multirow[t]{9}{*}{ SVM } & 94 & classified as & $\mathrm{a}$ & $b$ & a & 0.746 & 0.979 & 0.847 \\
\hline & & label a & 47 & 1 & $b$ & 0.970 & 0.667 & 0.790 \\
\hline & & label b & 16 & 32 & weighted average & 0.858 & 0.823 & 0.819 \\
\hline & {$[94,118]^{\top}$} & classified as & $a$ & $b$ & a & 0.787 & 1.000 & 0.881 \\
\hline & & label a & 48 & 0 & $b$ & 1.000 & 0.729 & 0.843 \\
\hline & & label b & 13 & 35 & weighted average & 0.893 & 0.865 & 0.862 \\
\hline & {$[94,118,93]^{T}$} & classified as & $\mathrm{a}$ & $b$ & $\mathrm{a}$ & 0.774 & 1.000 & 0.873 \\
\hline & & label a & 48 & 0 & $b$ & 1.000 & 0.708 & 0.829 \\
\hline & & label b & 14 & 34 & weighted average & 0.887 & 0.854 & 0.851 \\
\hline
\end{tabular}


tance, LDA, kNN, RF or SVM can be rightly assigned and is suitable for different sample distributions. Qualitative and quantitative experimental results have demonstrated the effectiveness of ECFS-DEA.

\section{Availability and requirements \\ Project name: ECFS-DEA \\ Project home page: http://bio-nefu.com/resource/ecfs- dea \\ Operating system(s): Linux, Windows, Mac \\ Programming language: Python $(\geq 3.5)$ \\ License: GPLv3 \\ Any restrictions to use by non-academics: none}

\begin{abstract}
Abbreviations
DEA: Differential expression analysis; ECFS-DEA: Ensemble classifier-based feature selection for differential expression analysis; JCD-DEA: Joint covariate detection for differential expression analysis; kNN: k-nearest-neighbor; LDA: Fisher's linear discriminative analysis; PBS: Portable batch system; RF: Random forest; ROC: Receiver operating characteristic; SAM: Significance analysis of microarrays
\end{abstract}

\section{Acknowledgements}

Not applicable.

\section{Authors' contributions}

XDZ and GHW conceived the general project and supervised it. QJ, HYL and YMW were the principal developers. QJ and HYL developed the main graphical user interface parts. HXW made the supplementary experiments on the simulated data. YMW wrote the client-server connection related part and the software documentation. HYL and YMW built the server components. QJ developed the underlying source code. XDZ wrote the original manuscript. SH tested the software and made the revision on the manuscript. All authors read and approved the final manuscript.

\section{Funding}

This work has been supported by the financial support of Natural Science Foundation of China (No. 61771165), China Postdoctoral Science Foundation Funded Project (No. 2014M551246, 2018T110302), Innovation Project of State Key Laboratory of Tree Genetics and Breeding (Northeast Forestry University, No.2019A04), Fundamental Research Funds for the Central Universities (No. 2572018BH01), National Undergraduate Innovation Project (No. 201910225184) and Specialized Personnel Start-up Grant (Also National Construction Plan of World-class Universities and First-class Disciplines, No. 41113237). The funding body of Natural Science Foundation of China played an important role in the design of the study, collection, analysis and interpretation of data and in writing the manuscript.

\section{Availability of data and materials}

The public dataset analysed during the current study is available in the GEO repository. GSE22058 is available at https://www.ncbi.nlm.nih.gov/geo/query/ acc.cgi?acc=GSE22058. The simulated data can be downloaded on http://bionefu.com/resource/ecfs-dea.

\section{Ethics approval and consent to participate}

Not applicable.

\section{Consent for publication}

Not applicable.

\section{Competing interests}

The authors declare that they have no competing interests.

\section{Author details}

${ }^{1}$ College of Information and Computer Engineering, Northeast Forestry University, No.26 Hexing Road, 150040 Harbin, China. ${ }^{2}$ Department of Neurology, The 2nd Affiliated Hospital of Harbin Medical University, No. 246
Xuefu Road, 150086 Harbin, China. ${ }^{3}$ State Key Laboratory of Tree Genetics and Breeding, Northeast Forestry University, No.26 Hexing Road, 150040 Harbin, China.

Received: 3 December 2019 Accepted: 27 January 2020

Published online: 05 February 2020

\section{References}

1. Lambrou Gl, Sdraka M, Koutsouris D. The "Gene Cube": a novel approach to three-dimensional clustering of gene expression data. Curr Bioinforma. 2019;14:721-7.

2. Storey JD, Tibshirani R, Garrett ES, Irizarry R, Zeger SL. SAM thresholding and false discovery rates for detecting differential gene expression in DNA microarrays. In: Parmigiani G, editor. The Analysis of, Gene Expression Data. Springer; 2003. p. 272-90. https://doi.org/10.1007/0387-21679-0_12.

3. Ritchie ME, Phipson B, Wu D, Hu Y, Law CW, Shi W, Smyth GK. Limma powers differential expression analyses for RNA-sequencing and microarray studies. Nucleic Acids Res. 2015;43:e47.

4. Pollard KS, Dudoit S, van der Laan MJ. Multiple testing procedures: the multiset package and application to genomics. In: Gentleman R, Carey V Huber W, Irizarry R, Dudoit S, editors. Bioinformatics and Computational Biology Solutions Using R and Bioconductor. Springer; 2005. p. 249-71. https://link.springer.com/chapter/10.1007\%2F0-387-29362-0_15.

5. Zhao XD, Wang L, Chen GS. Joint covariate detection on expression profles for identifying microRNAs related to venous metastasis in hepatocellular carcinoma. Sci Rep. 2017;7:1-11.

6. Kanji GK. 100 statistical tests, 3rd edition: SAGE Publication; 2006. https:// doi.org/10.4135/9781849208499.

7. Liu B, Gao X, Zhang H. BioSeq-Analysis2.0: an updated platform for analyzing DNA, RNA, and protein sequences at sequence level and residue level based on machine learning approaches. Nucleic Acids Res. 2019;47:e127.

8. Lai HY, Zhang ZY, Su ZD, Su W, Ding H, Chen W, Lin H. iProEP: a computational predictor for predicting promoter. Mol Ther Nucleic Acids. 2019;17:337-46.

9. Lv H, Zhang ZM, Li SH, Tan JX, Chen W, Lin H. Evaluation of different computational methods on 5-methylcytosine sites identification. Brief Bioinforma. 2019;bbz048:. https://academic.oup.com/bib/advancearticleabstract/doi/10.1093/bib/bbz048/5510088?redirectedFrom= fulltext.

10. Zou Q, Xing PW, Wei LY, Liu B. Gene2vec: gene subsequence embedding for prediction of mammalian n6-methyladenosine sites from mRNA. RNA. 2019;25:205-18.

11. Jia CZ, Zuo Y, Zou Q. O-GICNAcPRED-Il: an integrated classification algorithm for identifying O-GlcNAcylation sites based on fuzzy undersampling and a k-means PCA oversampling technique. Bioinformatics. 2018:34:2029-36.

12. Li SH, Zhang J, Zhao YW, Dao FY, Ding H, Chen W, Tang H. iPhoPred: a predictor for identifying phosphorylation sites in human protein. IEEE Access. 2019;7:17-28.

13. Lv ZB, Jin SS, Ding H, Zou Q. A random forest sub-Golgi protein classifier optimized via dipeptide and amino acid composition features. Front Bioeng Biotechnol. 2019;7:215.

14. Zhu XJ, Feng CQ, Lai HY, Chen W, Lin H. Predicting protein structural classes for low-similarity sequences by evaluating different features. Knowl Based Syst. 2019;163:787-93.

15. Ru XQ, Li LH, Zou Q. Incorporating distance-based top-n-gram and random forest to identify electron transport proteins. J Proteome Res. 2019;18:2931-9.

16. Li YJ, Niu MT, Zou Q. ELM-MHC: an improved MHC identification method with extreme learning machine algorithm. J Proteome Res. 2019;18: 1392-401.

17. Li C, Liu B. MotifCNN-fold: protein fold recognition based on fold-specific features extracted by motif-based convolutional neural networks. Brief Bioinforma. 2019;bbz133:. https://doi.org/10.1093/bib/bbz133.

18. Liu B, Zhu Y, Yan K. Fold-LTR-TCP: protein fold recognition based on triadic closure principle. Brief Bioinforma. 2019;bbz139:. https://doi.org/ 10.1093/bib/bbz139.

19. Liu B, Li C, Yan K. DeepSVM-fold: Protein fold recognition by combining Support Vector Machines and pairwise sequence similarity scores 
generated by deep learning networks. Brief Bioinforma. 2019;bbz098. https://doi.org/10.1093/bib/bbz098.

20. Song J, Wang H, Wang J, Leier A, Marquez-Lago T, Yang B, Zhang Z, Akutsu T, Webb Gl, Daly RJ. PhosphoPredict: A bioinformatics tool for prediction of human kinase-specific phosphorylation substrates and sites by integrating heterogeneous feature selection. Sci Rep. 2017;7:6862.

21. Song J, Tan H, Boyd SE, Shen H, Mahmood K, Webb Gl, Akutsu T, Whisstock JC, Pike RN. Bioinformatic approaches for predicting substrates of proteases. J Bioinforma Comput Biol. 2011;9:149-78.

22. Song J, Tan H, Wang M, Webb Gl, Akutsu T. TANGLE: two-level support vector regression approach for protein backbone torsion angle prediction from primary sequences. PloS ONE. 2012;7:e30361.

23. Cheng XP, Cai HM, Zhang Y, Xu B, Su WF. Optimal combination of feature selection and classification via local hyperplane based learning strategy. BMC Bioinformatics. 2015;16:219.

24. Cai HM, Ruan PY, Ng M, Akutsu T. Feature weight estimation for gene selection: a local hyperlinear learning approach. BMC Bioinformatics. 2014; $15: 70$.

25. Shmueli G. To Explain or to Predict? 2010;25:289-311. https://doi.org/10 2139/ssrn.1351252.

26. Breiman L. Random forests. Mach Learn. 2001;45:5-32.

27. Lv ZB, Jin SS, Ding H, Zou Q. A random forest sub-Golgi protein classifier optimized via dipeptide and amino acid composition features. Front Bioeng Biotechnol. 2019;7:215

28. Li Y, Liu YN, Wu YM, Zhao XD. JCD-DEA: a joint covariate detection tool for differential expression analysis on tumor expression profiles. BMC Bioinformatics. 2019;20:365.

29. Burchard J, Zhang C, Liu AM, Poon RT, Lee NPY, Wong KF, Sham PC, Lam BY, Ferguson MD, Tokiwa G, Smith R, Leeson B, Beard R, Lamb JR, Lim L, Mao M, Dai H, Luk JM. microRNA-122 as a regulator of mitochondrial metabolic gene network in hepatocellular carcinoma. Mol Syst Biol. 2010;24:402.

30. Ma JC, Qin CY, Yuan ZG, Liu SL. LncRNA PAPAS promotes hepatocellular carcinoma by interacting with miR-188-5p. J Cell Biochem. 2019;120: 13494-500.

31. Meng FZ, Zhang SG, Song RP, Liu Y, Wang JB, Liang YJ, Wang JZ, Han JH, Song X, Lu ZY, Yang GC, Pan SH, Li XY, Liu YF, Zhou F, Wang Y, Cui YF, Zhang B, MaK, Zhang CY, Sun YF, Xin MY, Liu LX. NCAPG2 overexpression promotes hepatocellular carcinoma proliferation and metastasis through activating the STAT3 and NF-kappa B/miR-188-3p pathways. Ebiomedicine. 2019;44:237-49.

\section{Publisher's Note}

Springer Nature remains neutral with regard to jurisdictional claims in published maps and institutional affiliations.

\section{Ready to submit your research? Choose BMC and benefit from:}

- fast, convenient online submission

- thorough peer review by experienced researchers in your field

- rapid publication on acceptance

- support for research data, including large and complex data types

- gold Open Access which fosters wider collaboration and increased citations

- maximum visibility for your research: over $100 \mathrm{M}$ website views per year

At $B M C$, research is always in progress.

Learn more biomedcentral.com/submissions 